\title{
Gender-specific associations between apolipoprotein A1 and arterial stiffness in patients with nonalcoholic fatty liver disease
}

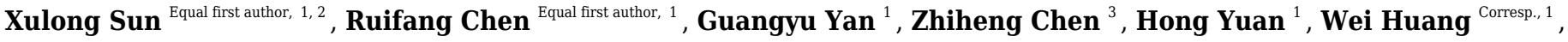 \\ Yao Lu ${ }^{\text {Corresp. } 1,4}$ \\ ${ }^{1}$ Clinical Research Center, The Third Xiangya Hospital of Central South University, Changsha, China \\ 2 \\ 2 Department of General Surgery, The Third Xiangya Hospital of Central South University, Changsha, China \\ 3 Health Management Center, The Third Xiangya Hospital of Central South University, Changsha, China \\ 4 Department of Life Science and Medicine, King's College London, London, United Kingdom \\ Corresponding Authors: Wei Huang, Yao Lu \\ Email address: 118312057@csu.edu.cn, yao.lu@kcl.ac.uk
}

Background: Lipid metabolism factors may play an important role in the progression of Nonalcoholic fatty liver disease (NAFLD) and its related cardiovascular dysfunctions. The study aims to assess whether Apolipoprotein A-1 (ApoA1) was associated with vascular stiffness in NAFLD patients.

Methods: From 2012-2013, we included 2,295 non-alcohol users with fatty liver disease (1,306 male patients) and completely excluded subjects who drank any alcohol ever to eliminate the effect of alcohol intake. The serum ApoA1 levels and the brachial-ankle pulse wave velocity (baPWV) were measured.

Results: Male's baPWV is much higher than female patients $(1412.79 \mathrm{~cm} / \mathrm{s}$ VS $1358.69 \mathrm{~cm} / \mathrm{s}, \mathrm{P}<0.001)$. ApoAl level was positively associated with baPWV (Odd Ratio(OR), 4.18; 95\% confidence interval (Cl), 1.16-15.1, $\mathrm{P}<0.05)$ in patients with AST/ALT $<1$ and $(\mathrm{OR}, 4.70 ; 95 \% \mathrm{Cl}, 1.36-16.23, \mathrm{P}<0.05)$ in patients with AST/ALT $\geq 1$ respectively. Only male's arterial stiffness is associated with ApoAl (OR, 3.96; $95 \% \mathrm{Cl}$, $1.29,12.30, \mathrm{P}<0.05)$ in logistics regression models adjusted for age, gender, body mass index(BMI), education attainment, physical activity, smoking, history of hypertension and high-density lipoprotein( $\mathrm{HDL}$ ). The relationship between ApoA1 and baPWV in male NAFLD patients remained significant (Confidence, $156.42 ; 95 \% \mathrm{Cl}, 49.34-263.50$. $\mathrm{P}<0.05$ ) in the fully adjusted linear regression model.

Conclusion: The serum ApoA1 was associated with arterial stiffness in male NAFLD patients. Increased ApoA1 level should be considered as an independent risk factor for arterial stiffness in male NAFLD patients, suggesting that NAFLD may alter arterial stiffness by "ApoA1-related" mechanism in men. 


\section{Gender-specific associations between Apolipoprotein}

3 A1 and arterial stiffness in patients with Nonalcoholic 4 Fatty Liver Disease

6 Xulong Sun ${ }^{1,2^{*}}$, Ruifang Chen ${ }^{1 *}$, Guangyu Yan ${ }^{1}$, Zhiheng Chen ${ }^{3}$, Hong Yuan ${ }^{1}$, Wei Huang ${ }^{1}$, Yao $7 \mathrm{Lu}^{1,4}$

${ }^{1}$ Clinical Research Center, The Third Xiangya Hospital of Central South University, Changsha, Hunan, China

${ }^{2}$ Department of General Surgery, The Third Xiangya Hospital of Central South University,

12 Changsha, Hunan, China

${ }^{3}$ Health Management Center, The Third Xiangya Hospital of Central South University,

14 Changsha, Hunan, China

${ }^{4}$ Department of Life Science and Medicine, King's College London, London, United Kingdom

*These authors contributed equally.

Corresponding Author:

Yao $\mathrm{Lu}^{1,4}$

the Third Xiangya Hospital of Central South University, 138 Tongzipo Road, Changsha, Hunan, 23 410013, China.

24 Email address: yao.lu@,kcl.ac.uk

25 Wei Huang ${ }^{1}$ 


\section{Abstract}

32 Background: Lipid metabolism factors may play an important role in the progression of

33

34

35

36

37

38

39

40

41

42

43

44

45 Nonalcoholic fatty liver disease (NAFLD) and its related cardiovascular dysfunctions. The study aims to assess whether Apolipoprotein A-1 (ApoA1) was associated with vascular stiffness in NAFLD patients.

Methods: From 2012-2013, we included 2,295 non-alcohol users with fatty liver disease (1,306 male patients) and completely excluded subjects who drank any alcohol ever to eliminate the effect of alcohol intake. The serum ApoA1 levels and the brachial-ankle pulse wave velocity (baPWV) were measured.

Results: Male's baPWV is much higher than female patients $(1412.79 \mathrm{~cm} / \mathrm{s}$ VS $1358.69 \mathrm{~cm} / \mathrm{s}$, $\mathrm{P}<0.001$ ). ApoA1 level was positively associated with baPWV (Odd Ratio(OR), 4.18; 95\% confidence interval $(\mathrm{Cl}), 1.16-15.1, \mathrm{P}<0.05)$ in patients with $\mathrm{AST} / \mathrm{ALT}<1$ and $(\mathrm{OR}, 4.70 ; 95 \% \mathrm{Cl}$, $1.36-16.23, P<0.05)$ in patients with $A S T / A L T \geqslant 1$ respectively. Only male's arterial stiffness is associated with ApoA1 (OR, 3.96; $95 \% \mathrm{Cl}, 1.29,12.30, \mathrm{P}<0.05)$ in logistics regression models adjusted for age, gender, body mass index(BMI), education attainment, physical activity, smoking, history of hypertension and high-density lipoprotein(HDL). The relationship between ApoA1 and baPWV in male NAFLD patients remained significant (Confidence, 156.42; $95 \% \mathrm{Cl}$, 49.34-263.50. $P<0.05$ ) in the fully adjusted linear regression model.

Conclusion: The serum ApoA1 was associated with arterial stiffness in male NAFLD patients. Increased ApoA1 level should be considered as an independent risk factor for arterial stiffness in male NAFLD patients, suggesting that NAFLD may alter arterial stiffness by "ApoA1-related" mechanism in men. 


\section{Introduction}

54 Nonalcoholic fatty liver disease (NAFLD) is increasingly recognized as a major chronic liver

55

56

57

58

59

60

61

62

63

64

65

66

67

68

69

70

71

72

73

74

75

76

77

78

79

80

81

82

83

84

85

86

87

88

89

90

91

disease not only in Western countries but also in Asia countries like China in which 15-30\% of the general adult population suffers from NAFLD (Wong 2013). NAFLD, which is characterized by the accumulation of triglycerides in hepatocytes in the absence of excessive alcohol intake and considered as systemic consequence(Byrne \& Targher 2015; Francque et al. 2016), has been confirmed to be associated with an increased risk of cardiovascular disease (CVD) and the incidence of cardiovascular events(De Alwis \& Day 2008). There is substantial evidence that NAFLD is associated with increased coronary artery calcification, increased pulse wave velocity (PWV), increased coronary membrane thickness, endothelial dysfunction, all of which are established markers of CVD (Fan et al. 2016; Iannaccone et al. 2007; Vlachopoulos et al. 2010) . More specifically, evidence NAFLD begins with the aberrant accumulation of triglyceride in the liver, thus lipid metabolism disorders play an important role in the disease progression as well as its complications like vascular dysfunction and related CVD outcomes (Francque et al. 2016) . Therefore, it is vital to identify the potential risk or biomarkers for the prevalence of cardiovascular diseases and related complications like arterial stiffness and vascular dysfunction, especially in NAFLD patients.

PWV is a valuable tool for assessing arterial stiffness by measuring the pulse propagation time and the travel distance between the studied arterial site, which is associated to abnormal vascular tone, thickening of smooth muscle and changes in blood viscosity(Deloach \& Townsend 2008; Kelly et al. 1989). Therefore, PWV is considered as a important marker of CVD events (Bae et al. 2013). Nevertheless, aortic stiffness modulation remains a strong target for therapeutic intervention, as it is the most powerful cardiovascular risk factor indeed a surrogate outcome rather than a risk factor.

Arterial stiffness could be progressive due to lipid metabolism (Fok \& Cruickshank 2015). Lipoprotein metabolism is usually thought to be associated with arterial changes, which will be of great interest in knowing how and to what extent arterial stiffness conditions are associated with CVD events in NAFLD patients (Sorokin \& Kotani 2015). Patients with NAFLD often have imbalances in lipid metabolism resulting in elevated very low lipoprotein and decreased highdensity lipoprotein (HDL) levels as well as low-density lipoprotein (LDL). Therefore, more lipid biomarkers should be identified to predict vascular dysfunctions. Recent studies forced on the effect HDL and LDL on arterial stiffness (Caldas et al. 2013; Gordon et al. 2013), but few concentrated on the protein components of these lipoproteins, especially in NAFLD patients. Apolipoprotein A-1 (ApoA1) is the major protein component of HDL, which plays an essential role in lipid production and in an important modulator of processes associated with glucose intolerance and diet-induced NAFLD development (Castellani \& Lusis 2001; Smith 2010). Lower ApoA1 has been reported to be statistically significant associations with high-risk atherosclerosis (Zhao et al. 2014). Nevertheless, the relationship between ApoA1 and PWV remains unknown.

Peer] reviewing PDF | (2020:04:48102:1:1:NEW 15 Jul 2020) 
92 In this study, we addressed the question whether the serum ApoA1 concentration was associated

93 with or was a risk factor for NAFLD. Whether ApoA1 was associated with arterial stiffness; and

94 also the relationship between ApoA1 and PWV in NAFLD were examined.

95 Materials \& Methods

96 People were recruited in the Health Management Center of The Third Xiangya Hospital from

97 March 2012 to December 2013. The study was approved by the Institutional Review Board of

98 Central South University (R18030) and informed oral consent was obtained from all subjects.

99 Evidence including non-alcoholic users NAFLD and NAFLD is based on the following items:(1)

100 The diffusion of near-field echoination in the hepatic region increases (stronger than that in the

101 kidney and spleen) and the far field echo is gradual attenuated;(2) Intra-hepatic lacuna structure

102 is not clear; (3) Mild to moderate hepatomegaly, with round and blunt border;(4) Color Doppler

103 ultrasound shows a decrease or even difficulty in showing blood flow signals in the liver, but the

104 distribution of blood flow is normal.If any one or more matches in items 1 and 2-4 are made, the

105 NAFLD is diagnosed. Hepatic ultrasound is carried out blindly by trained ultrasoundists (De

106 Zeng et al. 2008).

107 The exclusion criteria were as follows: 1) patients with hepatotoxic drug treatment; 2) history of

108 heart failure, stroke, arteriosclerosis, and diabetes; 3) participants with hepatitis C virus (HCV),

109 hepatitis B virus (HBV) and any chronic liver disease; 4) participants with other chronic

110 diseases: human immunodeficiency virus (HIV), cancer and end-stage kidney disease; 5)

111 incomplete data information.

112 Clinical and laboratory assessments

113 Anthropometric factors include height, weight, systolic blood pressure (SBP), diastolic blood

114 pressure (DBP) and the brachial-ankle pulse wave velocity (baPWV). Body mass index (BMI)

115 was calculated as weight $(\mathrm{kg})$ divided by height squared $\left(\mathrm{m}^{2}\right)$. Weight and height were measured

116 after participants had removed their shoes and heavy clothing. They were measured in meters

117 using a scale from the G-TECH Company. Blood pressure measurements were done with a

118 mercury sphygmomanometer with the participants in a chair using the right arm after $5 \mathrm{~min}$ in

119 rest. The average value of three readings was accepted as SBP and DBP.

120 Serum HDL, LDL and ApoA1 were prepared from fasting blood samples, and the supernatant

121 was collected and stored at $-20^{\circ} \mathrm{C}$ and $-80^{\circ} \mathrm{C}$. The serum levels of HDL, LDL were determined

122 by enzymatic methods and the Friedwald equation and ApoA1 was measured by an

123 immunological turbidity testing. They were measured using a biochemical detector from the

124 HITACHI Company (7600-020).

125 The definition of social behavior factors has also been reported in our previous study(Lu et al.

126 2015). Educational attainment is categorized as "< college graduation", "college graduation" or "

127 >college graduation". Physical activity and smoking had different types in the standard

128 questionnaires. Physical activity was categorized as "Never", "Occasionally" or "Frequently

129 (vigorous exercise at least three times per week)." Smoking was categorized as "Quit", "Never",

130 "Occasionally" or "Frequently". The diagnostic criteria of hypertension history were systolic or

131 diastolic blood pressure $\geqslant 140$ or $\geqslant 90 \mathrm{mmHg}$.

Peer] reviewing PDF | (2020:04:48102:1:1:NEW 15 Jul 2020) 


\section{2 baPWV measurement}

133 The brachial-ankle pulse wave velocity values were assessed using an automatic, non-invasive

134

135

136

137

138

139

140

141

142

143

144

145

146

147

148

149

150

151

152

153

154

155

156

157

158

159

160

161

162

163

164

165

166

167

168

169

170

171 vascular screening device Omron health medical (China) LTD, calculated as the distance travelled by the pulse wave divided by the time taken to travel the distance. The baPWV values were measured by the average of the left side baPWV and right side baPWV. The baPWV value is measured by the average of the baPWV on the left and the baPWV on the right. This is obtained by measuring blood pressure levels and pulsewaves in the arms and legs with four pneumatic pressure cuffs around the four limbs. Measurements were taken between 7:30 and 9:00 in the morning on the examination day. All the data was collected after the participants had 5 min of bed rest.

Since the reference value of baPWV up to $1400 \mathrm{~cm} / \mathrm{s}$ was used as an indicator of the severity of arteriosclerosis vascular damage, increased arterial stiffness was defined as baPWV of $1400 \mathrm{~cm} / \mathrm{s}$ or higher(Yamashina et al. 2003).

\section{Statistical Analysis}

Values for continuous variables are presented as mean \pm SD. Comparisons between female NAFLD patients and male NAFLD patients were performed using Pearson's Chi-square analysis for categorical variables and T-test for continuous variables. Multivariate logistic regression was used to assess the association between ApoA1 and baPWV totally and separately by gender. Linear regression analysis was used to determine the correlation between ApoA1 and baPWV in male NAFLD patients. The analysis was conducted after adjusted for age, gender, BMI, education, physical activity, smoking, history of hypertension, HDL and LDL level. As serum AST/ALT ratio is a good index to assess fibrosis advances. Patients can be divided into two groups by comparison of the ratio to 1 . Adjusted odds ratio and $95 \%$ confidence intervals (CIs) were calculated. An odds ratio $(\mathrm{OR})>1$ was considered a risk factor, and an $\mathrm{OR}<1$ was considered as a protective factor.

For exploratory purposes, the adjusted relationship between ApoA1 and the incidence of arterial stiffness was also assessed by a multivariate logistic regression model with restricted cubic splines. The relationships were depicted based on this model in which ApoA1 levels were categorized into $0.5 \mathrm{~g} / \mathrm{L}$ increment from $<1$ to $>3 \mathrm{~g} / \mathrm{L}$ and were adjusted for age, gender, BMI, education, physical activity, smoking, history of hypertension, HDL and LDL level. Values of $\mathrm{P}$ $<0.05$ were considered statistically significant. All the data analyses and figure were performed using STATA software version 14.0.

\section{Results}

A total of 2,295 subjects (1,306 males) were enrolled the baseline characteristics of the study participants grouped by gender are shown in Table 1. The participants' mean age was $48.47 \pm$ 10.22 years old, and the average BMI was $24.8 \pm 3.3 \mathrm{~kg} / \mathrm{m}^{2}$. The means of systolic and diastolic blood pressure were $124.65 \pm 16.54$ and $78.13 \pm 11.35 \mathrm{mmHg}$. The average HDL and LDL were $1.58 \pm 0.4 \mathrm{mmol} / \mathrm{L}$ and $3 \pm 0.88 \mathrm{mmol} / \mathrm{L}$. The means of ApoA 1 and baPWV were $1.51 \pm 0.28 \mathrm{~g} / \mathrm{L}$ and $1389.48 \pm 273.5 \mathrm{~cm} / \mathrm{s}$. There were significant differences in education attainment, smoking, and history of hypertension prevalence between male and female NAFLD patients $(\mathrm{P}<0.05)$. 
172 Female NAFLD patients are older and had lower BMI, blood pressure, baPWV and

173 predominantly had higher HDL and ApoA1 level $(\mathrm{P}<0.05$, Table 1$)$.

174 The total prevalence of arterial stiffness is $41.56 \%$, the prevalence for men is much higher than

175 that for women (44.79\% VS 35.39\%, P<0.001) (Table 1). A multi-logistic regression analysis

176 was performed to reveal the relationship between ApoA1 and baPWV among NAFLD patients

177 with different genders. The results of three different models were displayed in Table2. In model

178 1, after adjusted for age, gender and BMI, only ApoA1 level in male NAFLD patients was

179 significantly associated with baPWV (OR, 1.93; 95\% CI, 1.22-3.05. P<0.001). In model 2 which

180 adjusted for age, gender, BMI, education attainment, physical activity, smoking, and history of

181 hypertension, the overall ApoA1 level in NAFLD patients was positively associated with

182 baPWV (OR, 1.69; 95\% CI, 1.08-2.66. $\mathrm{P}<0.05)$; ApoA1 level in male NAFLD patients was

183 significantly associated with baPWV (OR, 2.80; 95\% CI, 1.55-5.04. P<0.001). No statistically

184 significant association was observed between female NAFLD patients' ApoA1 level and

185 baPWV. In model 3, adjusted for model 2 and HDL and LDL, the overall ApoA1 level in

186 NAFLD patients was significantly associated with baPWV (OR, 4.46; 95\% CI, 1.85-10.77.

$187 \mathrm{P}<0.001$ ); ApoA1 level in male NAFLD patients was significantly associated with baPWV (OR,

188 3.96; 95\% CI, 1.29-12.30. $\mathrm{P}<0.05$ ). No statistically significant association was observed between

189 ApoA1 and baPWV in female patients (Table 2). In the subgroups divided by serum AST/ALT

190 ratio, in model 1, only ApoA1 level in AST/ALT $<1$ subgroup was significantly associated with

191 baPWV (OR, 1.88; 95\% CI, 1.06-3.33. P<0.05). In model 3, the overall ApoA1 level in two

192 subgroups was positively associated with baPWV, (OR, 4.18; 95\% CI, 1.16-15.1. P<0.05) in

193 AST/ALT $<1$ subgroup and (OR, 4.70; 95\% CI, 1.36-16.23. $\mathrm{P}<0.05)$ in AST/ALT $\geqslant 1$ subgroup

194 respectively.

195 As shown in Table 3, the relationships between ApoA1 and baPWV in male NAFLD patients

196 were analyzed by linear regression. In model 1, the relationship between ApoA1 and baPWV in

197 male patients was found to be significant in univariate analysis (coefficient, 160.02; 95\% CI,

198 107.37-212.66. $\mathrm{P}<0.001$ ). In model 2, after adjusting for age, gender, BMI, education attainment,

199 physical activity, smoking and history of hypertension, the relationship between ApoA1 and

200 baPWV in male patients was statically significant (coefficient, 139.84; 95\% CI, 83.97-195.68.

$201 \mathrm{P}<0.001$ ). In model 3, which adjusted for model 2 and HDL and LDL, the relationship between

202 ApoA1 and baPWV in male NAFLD patients remained significant (coefficient, 156.42; 95\% CI,

203 49.34-263.50. $\mathrm{P}<0.05)$. ApoA1 is associated with the incidence of arterial stiffness only in male

204 NAFLD patients.

205 The relationship between ApoA1 and the incidence of arterial stiffness in male patients followed

206 a J-shaped curve, with increased odds ratio at low and high ApoA1. In a multivariable logistic

207 regression model, using ApoA1 as a covariate, adjusting for baseline covariates, the risk for the

208 incidence of arterial stiffness increased with ApoA1 (Figure 1).

209 Discussion

210 The major finding of this study is that risk of arterial stiffness increased with increasing ApoA1 
211 after adjusting for multi-confounders in NAFLD subjects. The relationship between arterial

212 stiffness and ApoA1 was statistically significant only in male NAFLD population.

213 The prevalence of NAFLD is likely to rise even further not only in western countries but also in

214 developing countries as China. There is growing evidence that NAFLD must be considered an

215 important independent risk factor for CVD(Ozturk et al. 2015). Longitudinal studies have shown

216 that cardiovascular mortality is the most important cause of death in patients with NAFLD(Byrne

217 et al. 2009). But the mechanisms by which NAFLD contributes to cardiovascular disease have

218 not yet been fully clarified. In our current study based on a mean age of 48.7 years old

219 population, the mean baPWV is $1389.48 \pm 273.5 \mathrm{~cm} / \mathrm{s}$, which is even much higher $(\mathrm{p}<0.001)$

220 than Chinese population with an equal age level $(\mathrm{p}=0.066)($ mean value: $1340 \mathrm{~cm} / \mathrm{s})$ (Zeng et al.

221 2016). NAFLD is associated with increased baPWV, thus it is of vital importance to identify the

222 risk factors for arterial stiffness in this specific population.

223 Hepatic steatosis arises from an imbalance between triglyceride (TG) acquisition and removal.

224 Many studies have demonstrated that hepatic TG accretion from increased dietary intake,

225 repartitioning of TG from adipose tissue to the liver, or increased de novo lipogenesis result in

226 hepatic steatosis(Berlanga et al. 2014; Cohen et al. 2011) [24,25]. ApoA1 is the major

227 apolipoprotein in HDL, which plays an important role in fatty acid metabolism(Yanai et al.

228 2015), thus ApoA1 can influence NAFLD by regulating the accumulation of liver triglyceride

229 intake in the $\operatorname{diet}($ Choe et al. 2013).ApoA1 plays a central role in the process of reverse

230 cholesterol transport (RCT), which has been thought to be the major mechanism of the

231 antiatherogenic functions of HDL(Yancey et al. 2003). In animal models, increasing HDL-C

232 levels did not affect the rates of RCT, and the rate of RCT achieved the maximum at the normal

233 levels of HDL-C(Alam et al. 2001). While infusion of a smaller ApoA1 containing into mice was

234 found to increase reverse cholesterol transport, and over-expression of ApoA1 was found to

235 favor efflux of cholesterol(Alam et al. 2001). At the same time, the liver is the major producers

236 of ApoA1 as well as the important location of reverse cholesterol transport(Timmins et al.

237 2005).Due to the limited studies on the relationship of ApoA1 and NAFLD, further research

238 should be conducted to clarify other potential mechanisms by which ApoA1 could affect the

239 pathologic processes of NAFLD.

240 According to previous studies, lower ApoA1 may have positive effect on atherosclerosis or high-

241 risk plaque features(Zhao et al. 2014), which is exactly in contradiction to our results. As there

242 are few publications focusing on the relationships between ApoA1 and PWV, unanimous

243 conclusions couldn't be drawn before we conducted more long-term large-scale clinical trials.

244 In our study on NAFLD subjects, men had significantly higher level of baPWV levels and

245 arterial stiffness than women, consistent with the results of some multicenter studies on the

246 general population(Kim et al. 2014). Many factors, such as body size, biochemical properties of

247 arteries, sex hormones, can lead to differences in arterial stiffness between male and

248 female(Janner et al. 2010; Rossi et al. 2011; Segers et al. 2007). Some studies showed the

249 distribution of ApoA1 differs between men and women, higher in women than in men (Csaszar

250 et al. 1991; Valles et al. 2002).A study of 147576 Swedish males and females aimed at ApoA1 
251 Distribution shown that the ApoA1 concentration was $10 \%$ lower than in females $(1.36 \pm 0.22 \mathrm{~g} / \mathrm{L}$

252 VS 1.51 $\pm 0.24 \mathrm{~g} / \mathrm{L}$ )(Jungner et al. 1998), which is consistent with the National Health and

253 Nutrition Examination Survey III of the United States(Bachorik et al. 1997). Therefore, we

254 investigated the relationships between arterial stiffness and ApoA1 in different gender group for

255 the first time. Female NAFLD subjects' serum ApoA1 level is much higher than Male's (1.62

256 VS 1.42, $\mathrm{P}<0.05$ ), which is exactly consistent with the results from other populations. The

257 difference in ApoA1 level in different gender groups implied that sex hormones may play an

258 important role in serum ApoA1 level. Both basic and bench research may be designed to test

259 hypothesis and the internal mechanisms.

\section{Limitation}

261

262

263

264

265

266

267

268

269

270

271

272

273

274

275

276

277

278

279

280

281

282

283

284

285

286

287

288

289

Fatty liver was diagnosed by ultrasonography rather than liver biopsy pathology. Liver biopsy is the "gold standard" for defining fat accumulation, inflammation and fibrosis in liver, but it is not suitable as a screening method for population-based epidemiological studies due to the invasiveness of liver biopsy. Additionally, this current study only investigated the relationships on NAFLD patients in a cross-section research, to clarify the impact of ApoA1 on CVD risks, further longitudinal study should be performed. Despite these limitations, this study conducted a meaningful study of the relationship between ApoA1 and arterial stiffness in NAFLD subjects.

\section{Conclusions}

Unlike the protective effect of ApoA1 on atherosclerosis, the serum ApoA1 was associated with arterial stiffness in male NAFLD patients, suggesting that NAFLD may alter arterial stiffness by "ApoA1-related" mechanism in male NAFLD population.

\section{Acknowledgements}

We are very thankful the suggestions on our work from Prof. Cruickshank Kennedy and Dr. Luca Faconti's in King's College London.

\section{References}

Alam K, Meidell RS, and Spady DK. 2001. Effect of up-regulating individual steps in the reverse cholesterol transport pathway on reverse cholesterol transport in normolipidemic mice. $J$ Biol Chem 276:15641-15649. 10.1074/jbc.M010230200

Bachorik PS, Lovejoy KL, Carroll MD, and Johnson CL. 1997. Apolipoprotein B and AI distributions in the United States, 1988-1991: results of the National Health and Nutrition Examination Survey III (NHANES III). 43:2364-2378.

Bae U, Shim J, Lee H, and Shin J. 2013. Serum carcinoembryonic antigen level is associated with arterial stiffness in healthy Korean adult. 415:286-289.

Berlanga A, Guiujurado E, Porras JA, and Auguet T. 2014. Molecular pathways in non-alcoholic fatty liver disease. 7:221-239.

Byrne CD, Olufadi R, Bruce KD, Cagampang FR, and Ahmed MH. 2009. Metabolic disturbances in non-alcoholic fatty liver disease. 116:539-564.

Byrne CD, and Targher G. 2015. NAFLD: A multisystem disease. 62. 
290

291

292

293

294

295

296

297

298

299

300

301

302

303

304

305

306

307

308

309

310

311

312

313

314

315

316

317

318

319

320

321

322

323

324

325

326

327

328

329

330

331

332

333

334

335

336

337

338

339

Caldas CAM, Borba EF, Bortolotto LA, Medeiros DM, Bonfa E, and Goncalves CR. 2013. Increased arterial stiffness assessed by pulse wave velocity in Behçet's disease and its association with the lipid profile. 27:454-459.

Castellani LW, and Lusis AJ. 2001. ApoA-II Versus ApoA-I Two for One Is Not Always a Good Deal. 21:1870-1872.

Choe YG, Jin W, Cho YK, Chung WG, Kim HJ, Jeon WK, and Kim BI. 2013. Apolipoprotein B/AI ratio is independently associated with non-alcoholic fatty liver disease in nondiabetic subjects. 28:678-683.

Cohen JC, Horton JD, and Hobbs HH. 2011. Human Fatty Liver Disease: Old Questions and New Insights. 332:1519-1523.

Csaszar A, Romics L, Karadi I, Tresch J, and Hansjurgen M. 1991. [Distribution of apolipoprotein A I and B in the blood according to age and gender, as well as their relation to blood cholesterol levels in the Hungarian blood donor population]. 132:1795.

De Alwis NMW, and Day CP. 2008. Non-alcoholic fatty liver disease: The mist gradually clears 48.

De Zeng M, Fan JG, Lu LG, Li Y, Chen C, Wang BY, and Mao YM. 2008. Guidelines for the diagnosis and treatment of nonalcoholic fatty liver diseases. 9:108-112.

Deloach S, and Townsend RR. 2008. Vascular Stiffness: Its Measurement and Significance for Epidemiologic and Outcome Studies. 3:184-192.

Fan Y, Wei F, Zhou Y, and Zhang H. 2016. Association of non - alcoholic fatty liver disease with impaired endothelial function by flow - mediated dilation: A meta - analysis. 46.

Fok H, and Cruickshank JK. 2015. Future Treatment of Hypertension: Shifting the Focus from Blood Pressure Lowering to Arterial Stiffness Modulation? 17:67-67.

Francque S, Der Graaff DV, and Kwanten WJ. 2016. Non-alcoholic fatty liver disease and cardiovascular risk: Pathophysiological mechanisms and implications. 65:425-443.

Gordon SM, Davidson WS, Urbina EM, Dolan LM, Heink A, Zang H, Lu LJ, and Shah AS. 2013. The Effects of Type 2 Diabetes on Lipoprotein Composition and Arterial Stiffness in Male Youth. 62:2958-2967.

lannaccone R, Piacentini F, Murakami T, Paradis V, Belghiti J, Hori M, Kim T, Durand F, Wakasa K, and Monden M. 2007. Hepatocellular carcinoma in patients with nonalcoholic fatty liver disease : Helical CT and MR imaging findings with clinical-pathologic comparison. 243:422-430.

Janner J, Godtfredsen NS, Ladelund S, Vestbo J, and Prescott E. 2010. Aortic augmentation index: reference values in a large unselected population by means of the SphygmoCor device. 23:180-185.

Jungner I, Marcovina SM, Walldius G, Holme I, Kolar W, and Steiner E. 1998. Apolipoprotein B and A-I values in 147576 Swedish males and females, standardized according to the World Health Organization-International Federation of Clinical Chemistry First International Reference Materials. Clin Chem 44:1641-1649.

Kelly RP, Hayward CS, Avolio A, and Orourke MF. 1989. Noninvasive determination of agerelated changes in the human arterial pulse. 80:1652-1659.

Kim JY, Park JB, Kim DS, Kim KS, Jeong JW, Park JC, Oh BH, and Chung N. 2014. Gender Difference in Arterial Stiffness in a Multicenter Cross-Sectional Study: The Korean Arterial Aging Study (KAAS). Pulse (Basel) 2:11-17. 10.1159/000365267

Lu Y, Lu M, Dai H, Yang P, Smithgagen J, Miao R, Zhong H, Chen R, Liu X, and Huang Z. 2015. Lifestyle and Risk of Hypertension: Follow-Up of a Young Pre-Hypertensive Cohort. 12:605-612.

Ozturk K, Uygun A, Guler AK, Demirci H, Ozdemir C, Cakir M, Sakin YS, Turker T, Sari S, and Demirbas S. 2015. Nonalcoholic fatty liver disease is an independent risk factor for atherosclerosis in young adult men. 240:380-386.

Peer) reviewing PDF | (2020:04:48102:1:1:NEW 15 Jul 2020) 
340

341

342

343

344

345

346

347

348

349

350

351

352

353

354

355

356

357

358

359

360

361

362

363

364

365

366

367

368

369

370

371

372

373

374

375

376
Rossi P, Frances Y, Kingwell BA, and Ahimastos AA. 2011. Gender differences in artery wall biomechanical properties throughout life. 29:1023-1033.

Segers P, Rietzschel E, De Buyzere M, Vermeersch S, De Bacquer D, Van Bortel LM, De Backer G, Gillebert TC, and Verdonck P. 2007. Noninvasive (Input) Impedance, Pulse Wave Velocity, and Wave Reflection in Healthy Middle-Aged Men and Women. 49:1248-1255.

Smith JD. 2010. Dysfunctional HDL as a Diagnostic and Therapeutic Target. 30:151-155.

Sorokin A, and Kotani K. 2015. Lipoprotein(a) and Arterial Stiffness Parameters. Pulse (Basel) 3:148-152. 10.1159/000438733

Timmins JM, Lee JY, Boudyguina E, Kluckman KD, Brunham LR, Mulya A, Gebre AK, Coutinho JM, Colvin PL, Smith TL, Hayden MR, Maeda N, and Parks JS. 2005. Targeted inactivation of hepatic Abca1 causes profound hypoalphalipoproteinemia and kidney hypercatabolism of apoA-I. J Clin Invest 115:1333-1342. 10.1172/jci23915

Valles V, Aguilarsalinas CA, Rez FJG, Rojas R, Franco A, Olaiz G, Rull JA, and Sepulveda J. 2002. Apolipoprotein $B$ and A-I distribution in Mexican urban adults: Results of a nationwide survey. 51:560-568.

Vlachopoulos C, Manesis EK, Baou K, Papatheodoridis GV, Koskinas J, Tiniakos D, Aznaouridis K, Archimandritis AJ, and Stefanadis C. 2010. Increased arterial stiffness and impaired endothelial function in nonalcoholic Fatty liver disease: a pilot study. 23:1183-1189.

Wong VW. 2013. Nonalcoholic fatty liver disease in Asia: A story of growth. 28:18-23.

Yamashina A, Tomiyama H, Arai T, Hirose K, Koji Y, Hirayama Y, Yamamoto Y, and Hori S. 2003. Brachial-Ankle Pulse Wave Velocity as a Marker of Atherosclerotic Vascular Damage and Cardiovascular Risk. 26:615-622.

Yanai H, Katsuyama H, Hamasaki H, Abe S, Tada N, and Sako A. 2015. Effects of Dietary Fat Intake on HDL Metabolism. 7:145-149.

Yancey PG, Bortnick AE, Kellner-Weibel G, de la Llera-Moya M, Phillips MC, and Rothblat GH. 2003. Importance of different pathways of cellular cholesterol efflux. Arterioscler Thromb Vasc Biol 23:712-719. 10.1161/01.Atv.0000057572.97137.Dd

Zeng Q, Dong SY, Wang M, Wang F, Li JM, and Zhao X. 2016. Cardiac autonomic neuropathy risk estimated by sudomotor function and arterial stiffness in Chinese subjects. 30:720725.

Zhao XQ, Hatsukami TS, Hippe DS, Sun J, Balu N, Isquith D, Crouse JR, Anderson TJ, Huston JI, and Polissar NL. 2014. Clinical Factors Associated With High-Risk Carotid Plaque Features as Assessed by Magnetic Resonance Imaging in Patients With Established Vascular Disease (from the AIM-HIGH Study). 114:1412-1419. 


\section{Table $\mathbf{1}$ (on next page)}

Table 1 Characteristics of the study participants, by gender

Notes: Note: Values for categorical variables are presented as number (percentage); Values for continuous variables are presented as mean \pm SD. 


\begin{tabular}{|c|c|c|c|c|c|c|c|}
\hline Variable & Total(me & $\mathrm{n} \pm \mathrm{SD})$ & \multicolumn{2}{|c|}{$N=1306$} & \multicolumn{2}{|c|}{$\mathrm{N}=989$} & $\begin{array}{l}\mathrm{P} \\
\text { value } \\
\square \\
\end{array}$ \\
\hline Age (y) & 48.47 & \pm 10.22 & 47.76 & 9.92 & 49.4 & +9.33 & 0.0001 \\
\hline BMI & 24.80 & \pm 3.3 & 25.57 & 3.14 & 23.79 & \pm 3.24 & $<0.001$ \\
\hline $\mathrm{SBP}(\mathrm{mmHg})$ & 124.65 & \pm 16.54 & 126.54 & 15.09 & 122.13 & 17.98 & $<0.001$ \\
\hline DBP (mmHg) & 78.13 & \pm 11.35 & 80.88 & 11.24 & 74.5 & \pm 10.45 & $<0.001$ \\
\hline \multicolumn{8}{|l|}{$\begin{array}{l}\text { Educational } \\
\text { attainment (\%) }\end{array}$} \\
\hline$<$ College graduation & 365 & $(15.9 \%)$ & 275 & $(21.1 \%)$ & 90 & $(9.1 \%)$ & \\
\hline College graduation & 1481 & $(64.5 \%)$ & 955 & $(73.1 \%)$ & 526 & $(53.2 \%)$ & $<0.001$ \\
\hline$>$ College graduation & 449 & $(19.6 \%)$ & 76 & $(5.8 \%)$ & 373 & $(37.7 \%)$ & \\
\hline \multicolumn{8}{|l|}{$\begin{array}{l}\text { Physical Activity } \\
(\%)\end{array}$} \\
\hline Never & 251 & $(10.9 \%)$ & 161 & $(12.3 \%)$ & 90 & $(9.1 \%)$ & \\
\hline Occasionally & 560 & $(24.4 \%)$ & 304 & $(23.3 \%)$ & 256 & $(25.9 \%)$ & \\
\hline Frequently & 1484 & $(64.7 \%)$ & 841 & $(64.4 \%)$ & 643 & $(65.0 \%)$ & 0.04 \\
\hline \multicolumn{8}{|l|}{ Smoking Status (\%) } \\
\hline Quit & 101 & $(4.4 \%)$ & 93 & $(7.1 \%)$ & 8 & $(0.8 \%)$ & \\
\hline Never & 1423 & $(62.0 \%)$ & 548 & $(42.0 \%)$ & 875 & $(88.5 \%)$ & \\
\hline Occasionally & 207 & $(9.0 \%)$ & 170 & $(13.0 \%)$ & 37 & $(3.7 \%)$ & $<0.001$ \\
\hline Frequently & 564 & $(24.6 \%)$ & 495 & $(37.9 \%)$ & 69 & $(7.0 \%)$ & \\
\hline $\begin{array}{l}\text { History of } \\
\text { Hypertension (\%) }\end{array}$ & 246 & $(10.7 \%)$ & 150 & $(11.5 \%)$ & 96 & $(9.7 \%)$ & $<0.001$ \\
\hline HDL(mmol/L) & 1.58 & \pm 0.4 & 1.43 & 0.35 & 1.77 & \pm 0.39 & $<0.001$ \\
\hline $\mathrm{LDL}(\mathrm{mmol} / \mathrm{L})$ & 3.00 & \pm 0.88 & 3.01 & 0.88 & 2.98 & \pm 0.89 & 0.54 \\
\hline ApoA1 (g/L) & 1.51 & \pm 0.28 & 1.43 & 0.26 & 1.62 & \pm 0.27 & $<0.001$ \\
\hline Right baPWV (cm/s) & 1388.01 & \pm 278.2 & 1412.51 & 261.31 & 1355.66 & 上 296.09 & \\
\hline Left baPWV $(\mathrm{cm} / \mathrm{s})$ & 1390.95 & \pm 277.03 & 1413.07 & 257.73 & 1361.72 & $=298.24$ & \\
\hline baPWV $(\mathrm{cm} / \mathrm{s})$ & 1389.48 & \pm 273.5 & 1412.79 & 255.13 & 1358.69 & t 293.34 & $<0.001$ \\
\hline Arterial Stiffness & 953 & $(41.56 \%)$ & 585 & $(44.79 \%)$ & 350 & $(35.39 \%)$ & $<0.001$ \\
\hline
\end{tabular}




\section{Table 2 (on next page)}

Odd ratios for the Incidence of arterial stiffness, by gender

Model1: logistic regression for the Incidence of arterial stiffness and ApoA1, and adjusted for age, gender and BMI for total population; age and BMI for Male and Female population;

Model2: Model 1 and adjusted for Educational attainment, Physical activity, Smoking status and history of hypertension;

Model3: Model 2 and adjusted for HDL LDL.

$*: p<0.05 ; * *: p<0.001$ 


\begin{tabular}{|c|c|c|c|c|c|c|c|c|}
\hline \multirow[t]{3}{*}{ Variable } & \multicolumn{5}{|c|}{ Total } & \multirow{2}{*}{\multicolumn{2}{|c|}{$\begin{array}{c}\text { Male } \\
\mathrm{N}=1306\end{array}$}} & \multirow{2}{*}{$\begin{array}{l}\text { Female } \\
\mathrm{N}=989\end{array}$} \\
\hline & & $\mathrm{N}=2295$ & & $\mathrm{ST} / \mathrm{ALT}<1$ & $\mathrm{AST} / \mathrm{ALT} \geq 1$ & & & \\
\hline & OR & $95 \% \mathrm{CI}$ & OR & $95 \% \mathrm{CI}$ & OR $95 \% \mathrm{CI}$ & OR & $95 \% \mathrm{CI}$ & OR $95 \% \mathrm{CI}$ \\
\hline Model 1 & 1.20 & $(0.84,1.72)$ & $1.88^{*}$ & $(1.06,3.33)$ & $1.36(0.83,2.22)$ & $1.93^{* *}$ & $*(1.22,3.05)$ & $0.57(0.31,1.03)$ \\
\hline Model 2 & $1.69 *$ & $(1.08,2.66)$ & 1.89 & $(0.95,3.77)$ & $1.80(0.97,3.37)$ & $2.80 * *$ & $*(1.55,5.04)$ & $0.74(0.35,1.58)$ \\
\hline Model 3 & $4.46^{* *}$ & $(1.85,10.77)$ & $4.18^{*}$ & $(1.16,15.1)$ & $4.70 *(1.36,16.23)$ & $3.96^{*}$ & $(1.29,12.30)$ & $2.92(0.63,13.59)$ \\
\hline
\end{tabular}

1 


\section{Table 3(on next page)}

Linear Regression between Apoa1 and baPWV in male patients

Model1: Linear regression for baPWV and ApoA1;

Model2: Model 1 and adjusted for age, BMI Educational attainment, Physical activity, Smoking status and history of hypertension;

Model3: Model 2 and adjusted for HDL LDL. 


\begin{tabular}{llll}
\hline Variable & Coefficient & $95 \%$ CI & P value \\
\hline Model 1 & 160.02 & $107.37,212.66$ & $<0.001$ \\
Model 2 & 139.84 & $83.97,195.68$ & $<0.001$ \\
Model 3 & 156.42 & $49.34,263,50$ & 0.004 \\
\hline
\end{tabular}




\section{Figure 1}

Relationship between ApoA1 as a continuous variable and risk of the incidence of arterial stiffness in male patients

Relationship between ApoA1 as a continuous variable and risk of the incidence of arterial stiffness in male patients. Results were obtained by multivariable logistic regression with restricted splines including ApoA1. ApoA1 levels were categorized into $0.5 \mathrm{~g} / \mathrm{L}$ increment from $<1$ to $>3 \mathrm{~g} / \mathrm{L}$ and were adjusted for age, gender, BMI, education, physical activity, smoking, history of hypertension, HDL and LDL level. The upper and lower $95 \% \mathrm{Cls}$ are denoted by dotted line. 


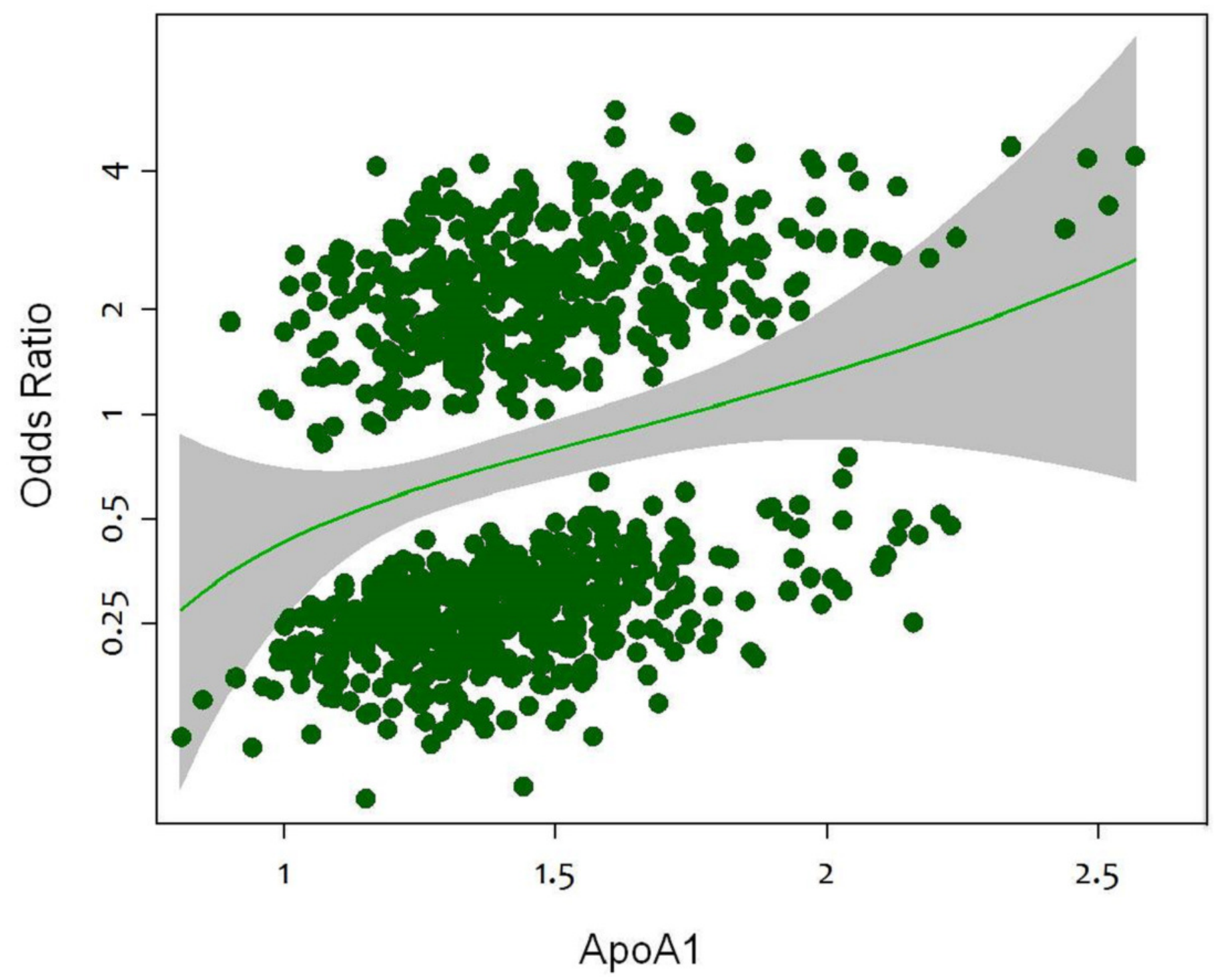

